\title{
Efeitos da disponibilidade de nitrogênio e fósforo no estabelecimento de Spartina alterniflora em um plano entremarés irregularmente alagado
}

\author{
Effects of nitrogen and phosphorus availability in the establishment of \\ Spartina alterniflora in an irregularly flooded intertidal flat
}

Paula Tassis de Mendonça ${ }^{1}$ paula_tassis@yahoo.com.br

César S.B. Costa ${ }^{2}$

costacsb@hotmail.com

Programa de Pós-Graduação em Oceanografia Biológica, FURG, Av. Itália, km 8 , Caixa Postal 474, 96201-900, Rio Grande, RS, Brazil.

${ }^{2}$ Departamento de Oceanografia, FURG, Av Itália, km 8, Caixa Postal 474, 96201-900, Rio Grande, RS, Brazil.

Resumo

Marismas são ambientes na zona entremarés, dominados por vegetação herbácea halófita, responsáveis por elevada produção primária e hábitats para vários invertebrados e peixes. No Rio Grande do Sul, as marismas vêm sofrendo crescentes impactos antrópicos e perdas cumulativas devido a processos erosivos naturais. Este estudo avaliou o processo de estabelecimento de uma nova marisma, a partir do plantio da grama Spartina alterniflora, sob diferentes tratamentos de adição de nutrientes, em uma praia do estuário da lagoa dos Patos (RS, Brasil). Propágulos vegetativos de S. alterniflora $(n=1113)$ foram transplantados com um espaçamento de $1 \mathrm{~m}$, em três blocos de plantio. As mudas foram submetidas a quatro tratamentos de adição de nutrientes: nitrogênio $\left(\mathrm{N}_{-}-\mathrm{NH}_{4}{ }^{+}\right)$, fósforo $\left(\mathrm{P}-\mathrm{PO}_{4}{ }^{-3}\right)$, nitrogênio e fósforo juntos (NP) e controle sem adição de nutrientes. Após quatro meses de plantio, plantas tratadas com P e NP apresentaram taxas de sobrevivência maiores (82\% e 87\%) do que as de plantas tratadas apenas com N $(70 \%)$ e as do controle sem nutrientes $(75 \%)$, sugerindo que o fósforo pode ser um fator estimulante da formação das raízes e do sucesso no estabelecimento. A adição conjunta de $\mathrm{N}$ e $\mathrm{P}$ resultou em aumentos médios de $143 \%$ no número de hastes por propágulo, $21 \%$ na altura das hastes e $75 \%$ da expansão horizontal dos rizomas, em relação às mudas do controle sem adição de nutrientes. Plantas no nível médio do bloco central de plantio, onde foi encontrada uma menor disponibilidade de fosfato, apresentaram o melhor desenvolvimento e tiveram seus crescimentos estimulados pela adição de fosfato. Esta resposta parece relacionada a uma densidade aumentada de aerênquima destas plantas grandes, que leva a oxidação da rizosfera.

Palavras-chave: Spartina, nutriente, respostas biogênicas, transplante. 
Paula T. de Mendonça, César S.B. Costa

and cumulative losses of their surface due to natural erosion. This study evaluated the process of establishing a new salt marsh by planting the grass Spartina alterniflora in a beach of the estuary of Patos Lagoon (RS), under different treatments for nutrient addition. Vegetative propagules of $S$. alterniflora $(n=1113)$ were planted spaced $1 \mathrm{~m}$ apart, inside 3 similar planting blocks. Propagules were divided among four groups treated with distinct nutrients: nitrogen $\left(\mathrm{N}_{-} \mathrm{NH}_{4}^{+}\right)$, phosphorus $\left(\mathrm{P}-\mathrm{PO}_{4}{ }^{-3}\right)$, a combination of nitrogen and phosphorus (NP) and control, without nutrient addition. After 4 months, plants treated with $\mathrm{P}$ and NP showed higher survivorship ( $82 \%$ e $87 \%$ ) than plants treated only with $\mathrm{N}(70 \%)$ and control plants $(75 \%)$, suggesting that phosphorus is a stimulating factor to root formation and is also responsible for the success in establishment. Propagules treated with combined $\mathrm{N}$ and $\mathrm{P}$ produced $143 \%$ more tiller, their tillers were $21 \%$ taller and rhizomes $75 \%$ longer than control propagules. The growth response of S. alterniflora at the addition of $\mathrm{N}$ and $\mathrm{P}$ showed spatial variation. Plants established in the central block of transplantation, where lower availability of soluble phosphate was found, showed the best development and had their growth increased by the addition of phosphate. This response seems to be related to an increased aerenchyma density of large S. alterniflora stand that may lead to rhiszosphere oxidation.

Key words: Spartina, nutrient, biogenic responses, transplantation.

\section{Introdução}

Marismas são ambientes dominados por vegetação herbácea halófita na zona entremarés; estão, portanto, sujeitas ao alagamento periódico e às variações de salinidade (Beeftink, 1977; Adam, 1990). Distribuídas mundialmente, em regiões tropicais (associadas a manguezais) e temperadas (Costa e Davy, 1992; Isacch et al., 2006), as marismas possuem elevada produção primária e contribuem tanto para o hábitat quanto para a alimentação de muitas espécies de invertebrados e peixes, entre as quais muitas com grande valor econômico (Adam, 1990, 2002; Costa et al., 1997). No Rio Grande do Sul, noventa e cinco por cento das marismas encontram-se no estuário da lagoa dos Patos (Coimbra e Costa, 2006). Atividades antrópicas como aterros, construção civil, despejo de efluentes domésticos e industriais, deposição de resíduos sólidos, agropecuária e aqüicultura têm causado a destruição e/ou contaminação dessas marismas nos últimos 150 anos (Seeliger e Costa, 1998). Nas últimas décadas, processos erosivos cumulativos das margens e ilhas do estuário da lagoa dos Patos têm causado também perdas significativas das marismas (Marangoni, 2008).
Frente ao cenário exposto, o plantio de novas áreas de marisma constitui uma alternativa viável de conservação desses ambientes. Desde a década de 1970, espécies da grama rizomatosa Spartina vêm sendo transplantadas em diversos países para recuperação de áreas sob o impacto da erosão costeira ou que foram degradadas por ação humana (Chung, 1982, 1993; Kruczynski, 1982; Knutson e Woodhouse, 1982; Lewis, 1982; Weeb, 1982; Woodhouse e Knutson, 1982; Matthews e Minelo, 1994). As marismas do estuário da lagoa dos Patos são dominadas por cinco espécies vegetais. Entre elas, a Spartina alterniflora recobre extensas áreas monoespecíficas de marismas abaixo do nível médio da água do estuário (Costa, 1998). Plantas do gênero Spartina possuem uma grande capacidade de trapear os sedimentos em suspensão, promovendo a deposição e prevenindo a erosão costeira (Broome et al., 1981; Chung, 1993; Pennings e Bertness, 2001). Espécies de Spartina são bioengenheiras e fundadoras que modelam as condições ambientais, reduzindo o estresse e facilitando a entrada de outras espécies nesses ambientes (Jones et al., 1994; Pennings e Bertness, 2001). Transplantes de plantas desse gênero podem ser feitos em peque- na, média ou larga escala, utilizando como unidade de propagação sementes ou material vegetativo, obtidos de ambientes naturais ou produzidos em viveiros (Kruczynski, 1982; Weeb, 1982; Woodhouse e Knutson, 1982; Costa, 2009).

No Brasil, ainda há poucos estudos sobre transplantes com plantas de marismas. O primeiro transplante piloto com $S$. alterniflora realizado no sul do Brasil utilizou 50 propágulos vegetativos e mostrou uma sobrevivência de $35 \%$ das plantas transplantadas, mas um rápido crescimento das plantas sobreviventes (Costa e Marangoni, 1997; Costa, 2009). Esses e outros estudos com $S$. alterniflora mostraram que a utilização de propágulos vegetativos (Copertino et al., 1997; Costa e Marangoni, 1997) e um espaçamento de 1-2 $\mathrm{m}$ entre as mudas aumentam o sucesso do transplante (Woodhouse e Knutson, 1982; Costa e Marangoni, 1997).

Vários fatores como tipo de sedimento, ação de ondas e ventos, disponibilidade de nutrientes, freqüência de alagamento e ação de pastadores influenciam no sucesso dos transplantes de Spartina (Lewis, 1982; Costa e Marangoni, 1997; Costa et al., 2003). O nitrogênio é o macroelemento que tem maior importância na produtivi- 
dade das marismas, atuando normalmente como fator limitante (Valiela e Teal, 1974; Cunha et al., 2005). Os solos pobres em oxigênio das marismas mais baixas na zona entremarés favorecem processos anaeróbicos denitrificadores, com perda de nitrogênio para a atmosfera (Valiela e Teal, 1979). Vários estudos em marismas euhalinas mostram que as plantas de $S$. alterniflora apresentam maior crescimento, quando fertilizadas com nitrogênio e, menos freqüentemente, com fósforo (Valiela e Teal, 1974; Broome et al., 1983; O'Brien e Zedler, 2006). Entretanto, a limitação do crescimento das plantas pelo fósforo é mais comum em marismas oligohalinas e em banhados de água doce, devido à grande disponibilidade de ferro e alumínio que podem precipitar com o fósforo sob condições oxidantes (Esteves, 1988; Odum, 1988)

A maioria dos estudos sobre respostas de crescimento de Spartina em função da disponibilidade de nutrientes foi realizada em áreas euhalinas sujeitas a macromarés regulares (Valiela e Teal, 1974; Broome et al., 1975; Chalmers, 1979; Pennings e Bertness, 2001), que suprem as mudas continuamente com água e nutrientes. Entretanto, pouco é conhecido sobre o papel dos macronutrientes no desenvolvimento de plantas em áreas oligo-mesohalinas e irregularmente alagadas por micromarés, como no sul do Rio Grande do Sul.

Para utilização de transplante da grama S. alterniflora como ferramenta para reverter o processo de perdas das áreas de marismas no estuário da lagoa dos Patos, as respostas de sobrevivência e de crescimento das plantas a diferentes condições edáficas da zona entremarés irregularmente inundada devem ser melhor compreendidas. Adicionalmente, o transplante de mudas para o ambiente natural pode ajudar na compreensão do processo de colonização da zona entremarés por plantas de marisma (Bertness, 1991; Pennings e Bertness, 2001; Costa et al., 2003) e dos fatores que determinam a formação de marismas no sul do Brasil. $\mathrm{O}$ objetivo deste trabalho foi avaliar o sucesso no estabelecimento de plantas de $S$. alterniflora, sob diferentes tratamentos de adição de nitrogênio $(\mathrm{N})$ e fósforo $(\mathrm{P})$, em um plano entremarés localizado no estuário irregularmente alagado da lagoa dos Patos (RS, Brasil).

\section{Material e métodos}

\section{Área de estudo}

O plantio de $S$. alterniflora foi desenvolvido na praia estuarina Lar Gaúcho $\left(32^{\circ} 02^{\prime} \mathrm{S}, 52^{\circ} 05^{\prime} \mathrm{W}\right)$, com cerca de $150 \mathrm{~m}$ de extensão (Figura 1), localizada na enseada semifechada do Saco da Mangueira, no estuário da lagoa dos Patos (Rio Grande, RS). Caracteristicamente micromareal (máxima amplitude de maré $\sim 0,5 \mathrm{~m}$ ) e irregularmente inundada (Costa et al., 1988; Costa et al., 1997), a praia está exposta a ventos do quadrante sul (SE-SW). Durante o período de estudo (20062007), a zona acima do nível médio da água na praia Lar Gaúcho encontrava-se fortemente degradada por depósito, no sedimento, de resíduos sólidos (plásticos, madeiras, entulhos de obras) de origem terrestre. Foi realizada a retirada de parte dos resíduos para o plantio de $S$. alterniflora. Nesse local, não existia vegetação antes do plantio.

Dados abióticos

A topografia na área de transplante foi medida, em outubro de 2006, a partir de um marco de concreto localizado na margem por meio de um nível laser (FPM Holding GMBH) e relacionada com o nível médio da Lagoa para a última década (1997-2006) (NML), estimado no ponto de monitoramento do programa de Pesquisa Ecológica de Longa Duração-PELD (www.peld. furg.br), localizado a $6 \mathrm{~km}$ do local de transplante. Foram coletadas três amostras de sedimento superficial $(0-5 \mathrm{~cm})$ do plano entremarés da praia Lar Gaúcho, para análise de granulometria (peneiramento e pipetagem) e estimativa da porcentagem de matéria orgânica (cinzamento a $550^{\circ} \mathrm{C}$ por $6 \mathrm{~h}$ ). Em fevereiro de 2007, foram coleta-

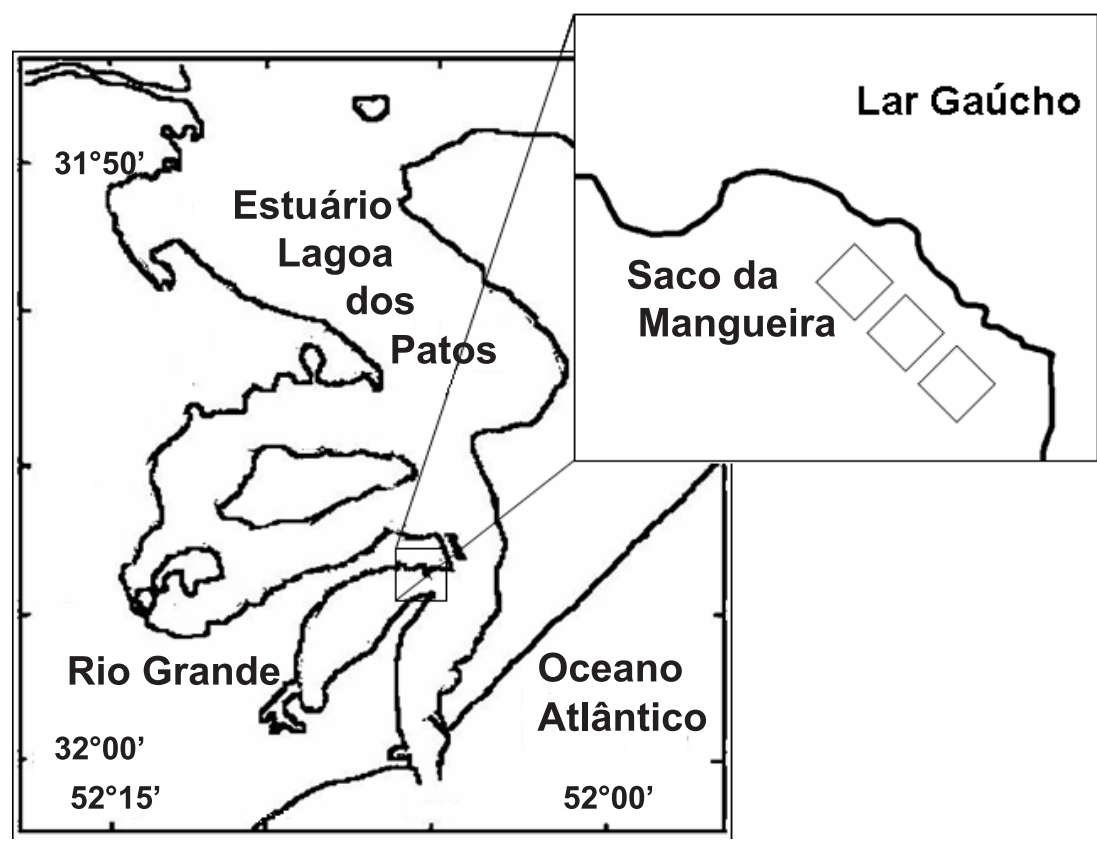

Figura 1. Localização da praia estuarina Lar Gaúcho no estuário da lagoa dos Patos, Rio Grande, RS.

Figure 1. Location of estuarine beach Lar Gaucho in the Patos Lagoon estuary, Rio Grande, RS. 
das 18 amostras de água intersticial, em covas rasas $(0-10 \mathrm{~cm})$ dentro dos blocos do transplante, cavadas durante período de exposição do plano entremarés. As amostras foram congeladas $\mathrm{e}$, posteriormente, analisadas quanto à concentração de fosfato $\left(\mathrm{P}-\mathrm{PO}^{-3}\right)$ pelo método descrito por Baumgarten et al. (1996). Adicionalmente, entre outubro de 2006 e fevereiro de 2007, foram registradas, diariamente, medidas de altura, salinidade e temperatura da água no ponto de monitoramento PELD (Costa et al., 2003). Valores de temperatura do ar foram obtidos na Estação Meteorológica da Fundação Universidade Federal de Rio Grande (http://www.meteorologia.furg.br).

Transplantes de mudas e sobrevivência global

Em setembro de 2006, propágulos vegetativos de $S$. alterniflora foram coletados em uma marisma localizada no lado oeste da ilha do Terrapleno, a cerca de $3 \mathrm{~km}$ do local de transplante. Essa marisma-fonte, originada de pequenos depósitos de dragado entre as décadas de 1950 e 1970, estava programada para ser removida pela autoridade portuária, para fornecer espaço necessário a operações de manobra de navios de grande porte no Porto Novo do Rio Grande (informação da Superintendência do Porto de Rio Grande - SUPRG). Os propágulos vegetativos coletados foram levados para uma casa de vegetação não climatizada, onde foram separados em mudas, contendo uma haste com raízes e fragmentos de rizomas. Essas mudas foram acomodadas em bandejas com areia fina e composto orgânico $(3: 1)$ para enraizamento, irrigadas, diariamente, com água de torneira. Em outubro de 2006, as mudas foram transplantadas com um espaçamento de $1 \mathrm{~m}$, dentro da zona entremarés da praia estuarina Lar Gaúcho, no Saco da Mangueira. O transplante foi efetuado em 3 blocos: dois eram de 20 x $20 \mathrm{~m}$ (21 x 21, colunas x linhas) e um de $10 \times 20 \mathrm{~m}$ (11 x 21, colunas x linhas), separados entre si por $3 \mathrm{~m}$ de plano arenolodoso (Figura 1). Um total de 1.113 mudas de $S$. alterniflo$r a$, recobrindo uma área de $1.120 \mathrm{~m}^{2}$ do plano arenolodoso entremarés da praia, foi utilizado para esse desenho experimental. A altura inicial de todas as hastes (da base do colmo ao topo da folha mais longa) foi medida com régua milimetrada e, semanalmente, durante o primeiro mês após o transplante, o número de hastes sobreviventes foi quantificado.

\section{Adição de nutrientes nas mudas transplantadas}

Após o primeiro mês do transplante, foram escolhidas aleatoriamente, na área transplantada, quatro grupos de 126 mudas sobreviventes, que foram posicionadas em um mapa de localização e marcadas com fio elétrico encapado. De forma a avaliar o efeito da disponibilidade de $\mathrm{N}$ e $\mathrm{P}$ no desenvolvimento inicial das mudas, elas foram submetidas a quatro tratamentos diferentes de adição nutrientes (granulado industrial, marca Ourofertil) junto à cova de plantio:

(i) $5 \mathrm{~g}$ de nitrogênio $\left(\mathrm{N}-\mathrm{NH}_{4}^{+}\right)$,

(ii) $5 \mathrm{~g}$ de fósforo $\left(\mathrm{P}^{-} \mathrm{PO}_{4}^{-3}\right)$,

(iii) $5 \mathrm{~g}$ de $\mathrm{N}$ e $5 \mathrm{~g}$ de $\mathrm{P}$ juntos,

(iv) controle, no qual não foi feita a adição de nutrientes.

No decorrer do texto, a partir desta enumeração, serão utilizadas as abreviações $\mathrm{N}, \mathrm{P}, \mathrm{NP}$ e $\mathrm{C}$ para os respectivos tratamentos. As formas de $\mathrm{N}$ e $\mathrm{P}$ utilizadas foram, respectivamente, sulfato de amônia e superfosfato. A aplicação dos nutrientes foi repetida a cada 30 dias durante três meses.

$\mathrm{O}$ número total de mudas marcadas sobreviventes foi monitorado com uma periodicidade mensal. Após três meses de adição de nutrientes, cada muda sobrevivente foi avaliada quanto ao número de hastes, à presença de estruturas reprodutivas (flores), à altura das hastes e à expansão horizontal dos rizomas (distância máxima de novas hastes em relação à haste inicialmente plantada).

\section{Análise dos dados}

Os dados foram analisados em relação ao tratamento de adição de nutrientes, aos blocos e ao nível do terreno do plantio. O levantamento topográfico do terreno demonstrou um desnível médio de $7 \mathrm{~cm}$, gradual entre a primeira e a última fileira dos três blocos de plantio. Para verificar a existência de relação entre a variação do nível do terreno com a sobrevivência global, após quatro semanas de plantio, foi feita uma Análise de Regressão Linear (Zar, 1984) entre a porcentagem de sobrevivência em cada fileira paralela a margem de cada bloco e os valores do nível do terreno destas fileiras. As mudas tratadas com N, P, NP e C tiveram as sobrevivências comparadas após quatro meses de plantio mediante a Análise de Qui-quadrado $\left(\chi^{2}\right)$ a 5\% de significância (Siegel, 1975).

Diferenças nas concentrações de fosfato na água intersticial entre diferentes blocos e níveis de plantio foram testadas por Análise de Variância (ANOVA) (Zar, 1984). Os valores de fosfato tiveram que ser logaritmizados antes da análise, de forma a atender os pré-requisitos de homocedasticidade e da normalidade (Zar, 1984).

Para testar as diferentes respostas de crescimento frente à adição de nutrientes, aos blocos transplantados e ao nível do terreno, foi feita Análise de Variância (Zar, 1984). O tratamento nível do terreno foi caracterizado por três classes topográficas; as mudas foram, classificadas entre os níveis de plantio superior, médio e inferior, respectivamente, quando plantadas de $0-2,0 \mathrm{~cm}, 2,1-4,0 \mathrm{~cm}$ e $4,1-7,0 \mathrm{~cm}$ abaixo do ponto mais alto do plantio. A altura inicial das hastes, medida em outubro de 2006, foi utilizada como co-variável nas ANOVAs, de forma a testar algum efeito do tamanho inicial da muda no desenvolvimento posterior das plantas. Os diferentes tratamentos de nutrientes, blocos de transplante e nível do terreno foram incluídos nas ANOVAs como variáveis independentes. As análises de variância foram 
utilizadas para interpretar as significâncias dos tratamentos sobre as variáveis bióticas (número de hastes, altura das hastes e expansão horizontal dos rizomas), bem como para estimar a quantidade da variação explicada em cada tratamento no desempenho das plantas, considerando a Soma dos Quadrados Totais como estimativa da variação total (Zar, 1984). De forma a atender os pré-requisitos de normalidade e homocedasticidade das ANOVAs, a transformação $\log _{10}$ (variável) foi utilizada para as variáveis número de hastes e altura das hastes, bem como a transformação raiz quadrada (variável), para expansão horizontal dos rizomas. Quando diferenças significativas foram detectadas na ANOVA, comparações entre níveis dos tratamentos foram analisadas com Teste de Tukey para tamanhos desiguais de amostras, ao nível de significância de 5\% (Zar, 1984).

Possíveis efeitos dos tratamentos de adição de nutrientes, blocos transplantados e nível do terreno sobre a floração de $S$. alterniflora foram testados mediante a Análise de Qui-quadrado $\left(\chi^{2}\right)$ das freqüências de hastes que floresceram a $5 \%$ de significância (Siegel, 1975).

Resultados

Fatores abióticos

O nível médio da lagoa dos Patos observado no período de estudo (24/10/2006-28/02/2007) foi de -18 $\pm 15 \mathrm{~cm}$ NML ( \pm desvio-padrão) (Figura 2). O local do transplante apresentou uma altura topográfica média de $-24 \mathrm{~cm}$ NML com uma amplitude de $7 \mathrm{~cm}$ entre o limite continental e o corpo d'água. Os níveis mais altos e mais baixos da área de plantio ficaram alagados, respectivamente, $60 \%$ e $79 \%$. Durante o período de estudo, a temperatura do ar variou entre $17,1^{\circ} \mathrm{C}$ e $31,4^{\circ} \mathrm{C}$, a temperatura média da água foi de $25,0 \pm 2,5^{\circ} \mathrm{C}$ e a salinidade média foi de $19,0 \pm 7,9$ $\mathrm{gNaCl} \mathrm{L}{ }^{-1}$.

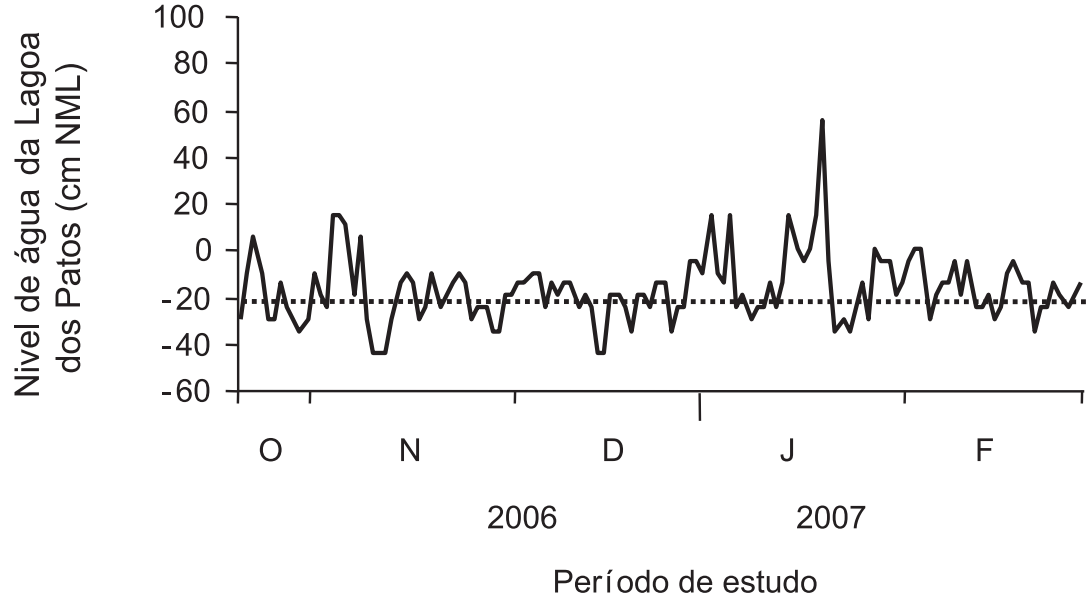

Figura 2. Variação diária do nível de água do estuário da lagoa dos Patos (RS) entre $24 / 10 / 2006$ a 28/02/2007. Linha tracejada representa o nível topográfico médio $(-24 \mathrm{~cm}$ $\mathrm{NML}$ ) da área transplantada com S. alterniflora. Abreviação: NML, nível médio decadal da lagoa (1997-2006).

Figure 2. Daily variation in water level of the Patos Lagoon estuary (RS) between $24 / 10 / 2006$ and $28 / 02 / 2007$. Dashed line represents the average topographic level (-24 $\mathrm{cm} \mathrm{NML)}$ of the area planted with S. alterniflora. Abbreviation: NML, average decadal water level of the lagoon (1997-2006).

A granulometria do sedimento do plano entremarés estuarino onde foi realizado o transplante não variou entre os blocos $(p>0,50)$, caracterizandose como predominantemente areia fina $(61,4 \%)$, associada à areia muito fina $(26,2 \%)$ (Figura 3A). O sedimento apresentou um baixo teor médio de finos (silte e argila; $5,5 \pm 3,2 \%$ ) e de matéria orgânica $(1,7 \pm 0,6 \%)$. Valores de fosfato na água intersticial, em fevereiro de 2007, variaram de $0,9-7,5 \mu \mathrm{M}$. Não foram encontradas diferenças significativas entre blocos $\left(\mathrm{F}_{\text {bloco }}=0,0 ; \mathrm{p}=0,97\right)$, mas a concentração média de fosfato no nível médio de plantio $(1,7 \pm 0,2 \mu \mathrm{M})$ foi significativamente $\left(\mathrm{F}_{\text {nível }}=4,3 ; \mathrm{p}<0,05\right)$ menor do que a do nível baixo $(3,8 \pm$ $1,1 \mu \mathrm{M})$ (Figura 3B).

Do total de 1.113 mudas transplantadas para a enseada no Saco da Mangueira, $87 \%$ e $80 \%$ sobreviveram, respectivamente, após duas e quatro semanas. Apesar de a sobrevivência ter variado de $66 \%$ a $85 \%$ entre os diferentes níveis do terreno e de $76 \%$ a $82 \%$ entre os blocos de plantio após quatro semanas, não foi observada diferença significativa na sobrevivência de mudas entre os níveis do terreno e os blocos de plantio $(\mathrm{r}$ Pearson $=0,1)$ (Figura 4). No primeiro mês após o plantio, as principais causas de morte foram a retirada das mudas por crianças da comunidade local, pisoteio por animais (cachorros, cavalos, etc.) e a perturbação por ondas e fortes ventos, em ocasiões de subida do nível da água. As causas de morte não foram quantificadas individualmente.

\section{Experimento de adição de nutrientes}

Após três meses de adição de nutrientes, a sobrevivência das plantas de $S$. alterniflora no LG não variou significativamente entre tratamentos de adição de nutrientes $\left(\chi^{2}=2,9, \mathrm{GL}=3\right.$, $\mathrm{p}=0,50)$. As plantas tratadas com NP, $\mathrm{P}$, controle e $\mathrm{N}$ apresentaram, respectivamente, taxas de sobrevivência de $88,84,75$ e $72 \%$ (Figura 5).

Após quatro meses do transplante, as maiores diferenças médias entre plantas dos tratamentos foram observadas no número de hastes produzidas por mudas sobreviventes e na máxima expansão horizontal dos rizomas; já as diferenças menos marcantes foram observadas na altura máxima das hastes. 

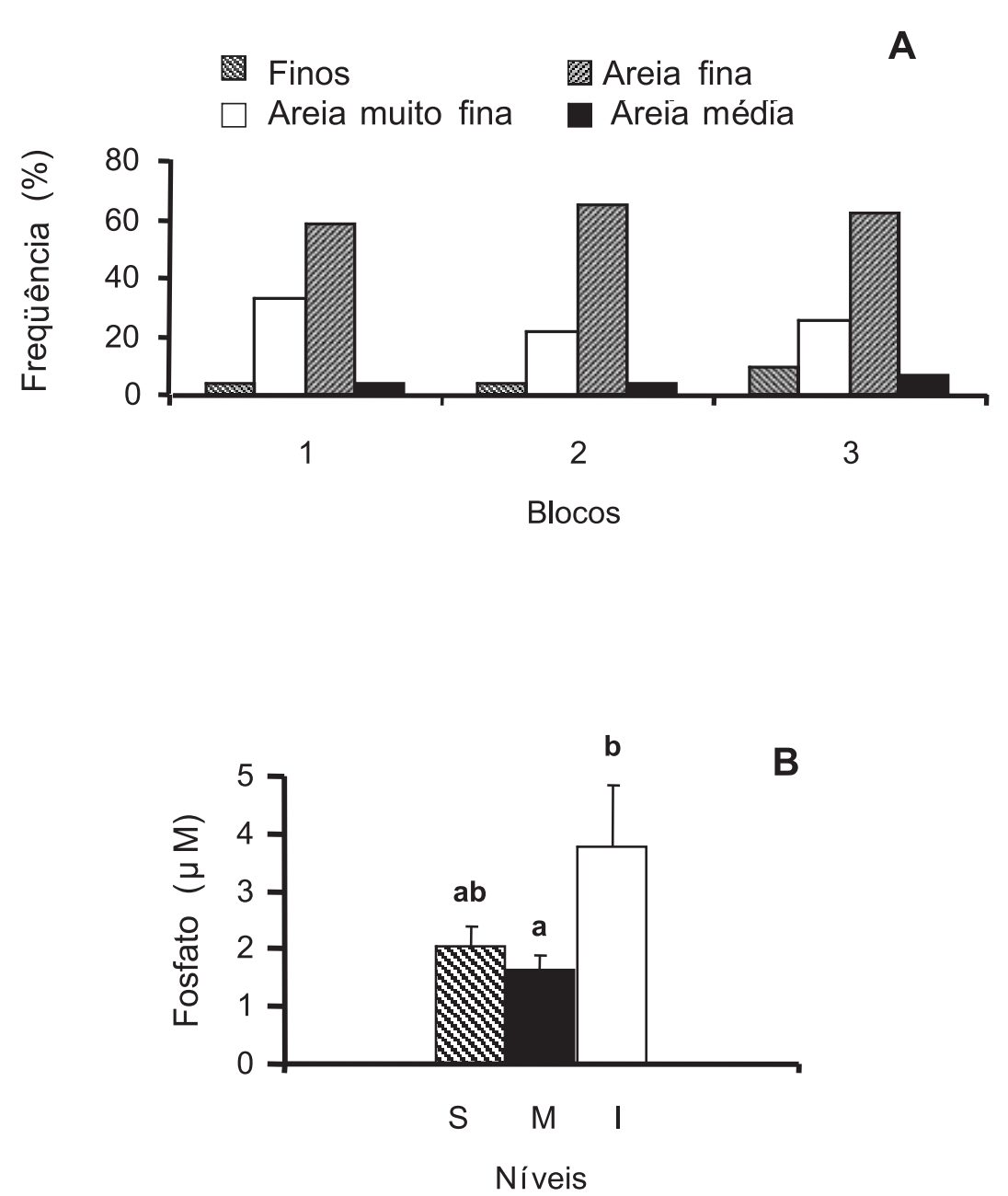

Figura 3. Granulometria e teor de matéria orgânica (\%) do sedimento superficial (0-5 $\mathrm{cm})(\mathbf{A})$ e concentrações de fosfato na água intersticial (B) em diferentes blocos e níveis de plantio de $S$. alterniflora no estuário da lagoa dos Patos. Diferentes letras minúsculas indicam médias significativamente (Teste de Tukey, $p<0,05$ ) entre níveis dos tratamentos. Abreviações: S, nível superior; $M$, nível médio; I, nível inferior.

Figure 3. Sediment grain size and organic matter content (\%) of surface sediment $(0-5 \mathrm{~cm})$ (A) and porewater phosphate concentration (B) at different intertidal levels and planting blocks of S. alterniflora in the Patos Lagoon estuary. Different lower case letters indicate significant differences (Tukey test, $p<0.05$ ) between treatment levels. Abbreviations: $\mathrm{S}$, upper level; M, medium level; I, lower level.

O número de hastes produzidas por mudas sobreviventes, a altura máxima das hastes e a máxima expansão horizontal dos rizomas foram diretamente relacionadas, de forma significativa $(\mathrm{p}$ $<0,05)$, ao tamanho inicial das mudas (estimada pela co-variável altura inicial) (Tabela 1). A adição de nutrientes e a posição dos blocos de transplantes explicaram de forma significativa $(\mathrm{p}<$ $0,001)$ a variabilidade da altura máxima das hastes (respectivamente, 7,4\% e $6,5 \%$ da variação total) e do número de hastes por muda (respectivamente $26,7 \%$ e $1,4 \%$ ). A variação da máxima expansão horizontal ocorreu, principalmente, devido à adição de nutrientes, que explicou $7,2 \%$ da variabilidade (Tabela 1). O nível do terreno também afetou significativamente ( $p$ $<0,05)$ todas as variáveis citadas acima, mas de forma pouco marcada.

Após quatro meses do transplante, as plantas tratadas com NP apresentaram um número expressivamente maior de hastes produzidas por muda $(\mathrm{p}<0,001)$, com uma média ( \pm erro-padrão) de 20,8 $\pm 1,7$ hastes significativamente maior do que as plantas tratadas com $\mathrm{P}(10,8$ $\pm 0,9$ hastes $)$ e as do controle $(8,5 \pm 0,7$ hastes) (Figura 6A). Adicionalmente, o número médio de hastes foi diferente entre os tratamentos NP e N nos blocos de transplante 1 e 3 , mas não no bloco 2 (central) (Figura 7A). No bloco 2, o número de hastes por muda submetidas aos tratamentos N e NP não foram significativamente diferentes entre si, bem como as plantas tratadas com $\mathrm{N}$ e isoladamente com $\mathrm{P}$ apresentaram um número expressivamente maior de hastes produzidas por muda do que as tratadas com os mesmos elementos no bloco 1 e 3. Essas diferenças parecem explicar a significância da interação Nutrientes $\mathrm{X}$ Blocos na ANOVA (Tabela 1).

As plantas tratadas com NP apresentaram máxima expansão horizontal média de $29,1 \pm 2,8 \mathrm{~cm}$, significativamente maior $(\mathrm{p}<0,001)$ do que as tratadas com $\mathrm{N}(20,6 \pm 2,5 \mathrm{~cm}), \mathrm{C}(16,8$ $\pm 1,9 \mathrm{~cm})$ e $\mathrm{P}(17,7 \pm 2,2 \mathrm{~cm})$ (Figura $6 B)$. Não foi observada interação entre o tratamento com nutrientes e os blocos de plantio para a expansão horizontal $(F=1,0 ; p<0,5)$ (Tabela 1$)$. No final do estudo, as plantas tratadas com NP apresentaram altura máxima média de $73,1 \pm 2,8 \mathrm{~cm}$, significativamente maior $(\mathrm{p}<0,001)$ do que dos demais tratamentos (médias, $\mathrm{C}=60,2$ $\pm 2,8 \mathrm{~cm} ; \mathrm{P}=57,8 \pm 2,9 \mathrm{~cm} ;$ e $\mathrm{N}=$ $57,2 \pm 2,9 \mathrm{~cm}$ ), que não se diferenciam (Figura 6C). A resposta de crescimento em altura variou significativamente entre blocos $\left(\mathrm{F}_{\text {Nutriente } \mathrm{X} \text { Blocos }}=5,0\right.$; $\mathrm{p}<0,001)$. Nos blocos 1 e 3 , as plantas tratadas com NP apresentaram uma altura máxima expressivamente maior do que as plantas tratadas isoladamente com N (Tabela 1; Figura 7C).

Independente da adição de nutrientes, as médias do número de hastes produzidas por muda e da altura máxima das hastes foram significativamente $(\mathrm{p}<0,05)$ maiores no bloco 2 , com valores de, respectivamente, $15,0 \pm 0,6$ hastes e $69,9 \pm$ 1,3 cm (Tabela 1; Figura 7).

Para as três respostas de crescimento, ocorreram diferenças significativas 
Tabela 1. Resultados das Análises de Variância para número de hastes produzidas, altura máxima das hastes e expansão horizontal máxima de rizomas de S. alterniflora, após quatro meses de plantio e tratamento de adição de nutrientes, em diferentes níveis do terreno e blocos de plantio no estuário da lagoa dos Patos (RS).

Table 1. Analyses of Variance results for the number of produced tillers, maximum tiller height and maximum horizontal expansion of rhizomes of S. alterniflora, after 4 months of planting and nutrient additions at different intertidal levels and planting blocks in Patos Lagoon estuary (RS).

\begin{tabular}{|c|c|c|c|c|c|c|c|c|c|c|c|c|c|}
\hline \multirow[t]{2}{*}{ Tratamento } & \multicolumn{5}{|c|}{ Número de hastes ${ }^{\# 1}$} & \multicolumn{4}{|c|}{ Expansão horizontal \#2 } & \multicolumn{4}{|c|}{ Altura das hastes ${ }^{\# 1}$} \\
\hline & GL & ss & $\mathbf{F}$ & & SS $\%$ & SS & $\mathbf{F}$ & & SS\% & ss & $\mathbf{F}$ & & SS $\%$ \\
\hline Altura inicial & 1 & 84,9 & 150,4 & $* * *$ & 18,6 & 7,6 & 65,2 & $* * *$ & 12,9 & 0,7 & 51,0 & $* * *$ & 9,4 \\
\hline Nutrientes & 3 & 122,0 & 72,0 & $* * *$ & 26,7 & 4,3 & 12,2 & $* * *$ & 7,2 & 0,5 & 13,3 & $* * *$ & 7,4 \\
\hline Blocos & 2 & 6,4 & 5,6 & * & 1,4 & 0,6 & 2,6 & & 1,0 & 0,5 & 17,5 & $* * *$ & 6,5 \\
\hline Nível & 2 & 7,2 & 6,4 & ** & 1,6 & 1,4 & 5,9 & * & 2,3 & 0,1 & 4,8 & * & 1,8 \\
\hline Nutrientes*Blocos & 6 & 16,9 & 5,0 & $* * *$ & 3,7 & 0,7 & 1,0 & & 1,1 & 0,4 & 5,0 & $* * *$ & 5,6 \\
\hline BlocosNível & 4 & 8,0 & 3,5 & * & 3,5 & 1,2 & 2,5 & * & 2,0 & 0,0 & 0,3 & & 0,2 \\
\hline Nutrientes*Nível & 6 & 2,7 & 0,8 & & 0,8 & 0,8 & 1,2 & & 1,4 & 0,1 & 1,0 & & 1,1 \\
\hline Resíduo & 369 & 208,4 & & & & 42,7 & & & & 4,7 & & & \\
\hline
\end{tabular}

\#1 log10 (variável)

\#2 raiz quadrada (variável)

* Significante a $p<0,05$

** Significante a $p<0,01$

${ }^{* * *}$ Significante a $p<0,001$

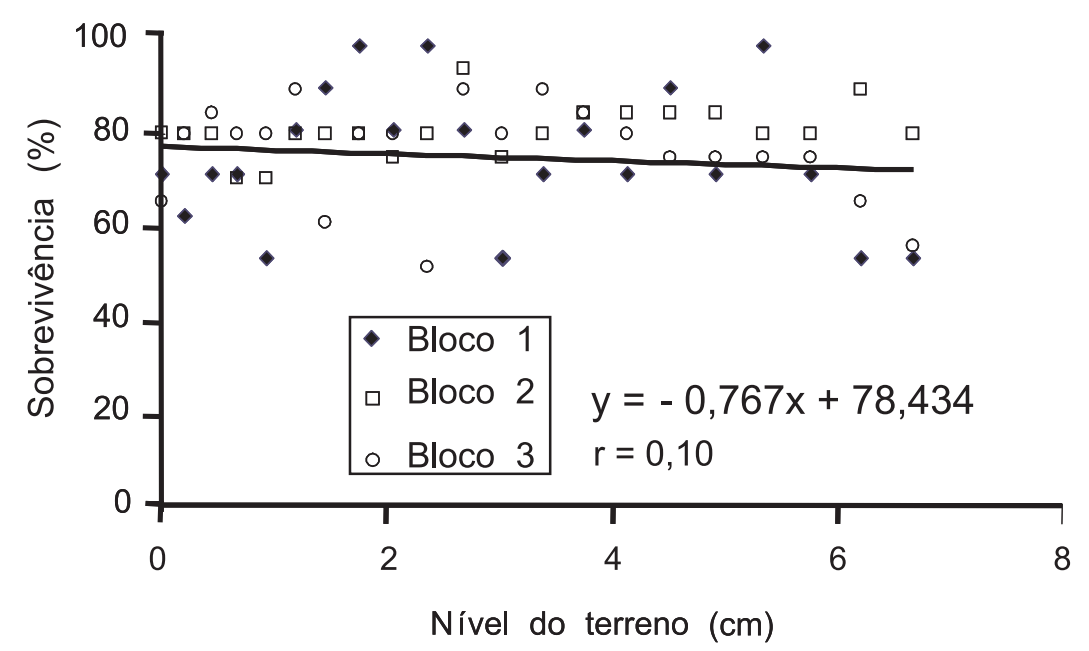

Figura 4. Análise de regressão linear entre sobrevivência global e o nível do terreno, após quatro meses de plantio das mudas de $S$. alterniflora no estuário da lagoa dos Patos (RS). Figure 4. Linear regression analysis between overall survival and intertidal level four months after transplantation of S. alterniflora in the Patos Lagoon estuary (RS).

entre os níveis de plantio $(\mathrm{p}<0,05)$ (Tabela 1; Figura 8). Para todos os parâmetros, o melhor desempenho ocorreu no nível médio; e os menores desempenhos, nos níveis inferior (para número de hastes e expansão horizontal) ou superior (para altura máxima das hastes) (Figuras 8A-C). Entretanto, essas diferenças não foram consistentes entre os blocos para número de hastes $\left(\mathrm{F}_{\text {Bloco } \mathrm{x} \text { Nivel }}=3,5\right.$; $\mathrm{p}<0,05)$ e para expansão dos rizomas
$\left(\mathrm{F}_{\text {Bloco X Nivel }}=2,5 ; \mathrm{p}<0,05\right)$ (Figuras 9A-C). De forma geral, nos blocos $1 \mathrm{e}$ 3 , um menor desempenho foi observado no nível inferior do plantio, entretanto, no bloco central, esse fato não foi observado.

Não foi constatada diferença significativa na porcentagem de hastes que floresceram entre os tratamentos com nutrientes $\left(\chi^{2}=5,12, \mathrm{GL}=3\right.$, $\mathrm{p}<0,16$ ), que variou de $46 \%$ a $70 \%$ (Figura 6D). Foi observada diferença expressiva entre os blocos de plantio na proporção de hastes que floresce$\operatorname{ram}\left(\chi^{2}=12,2, \mathrm{GL}=2, \mathrm{p}<0,01\right)$, com maior proporção no bloco $2(80 \%)$ (Figura 7D). Não se verificou diferença significativa na proporção de hastes que floresceram nos diferentes níveis do terreno $\left(\chi^{2}=0,59, \mathrm{GL}=2, \mathrm{p}<0,5\right)$ (Figuras 8D, 9D).

\section{Discussão}

As mudas de $S$. alterniflora transplantadas para zona entremarés de uma 


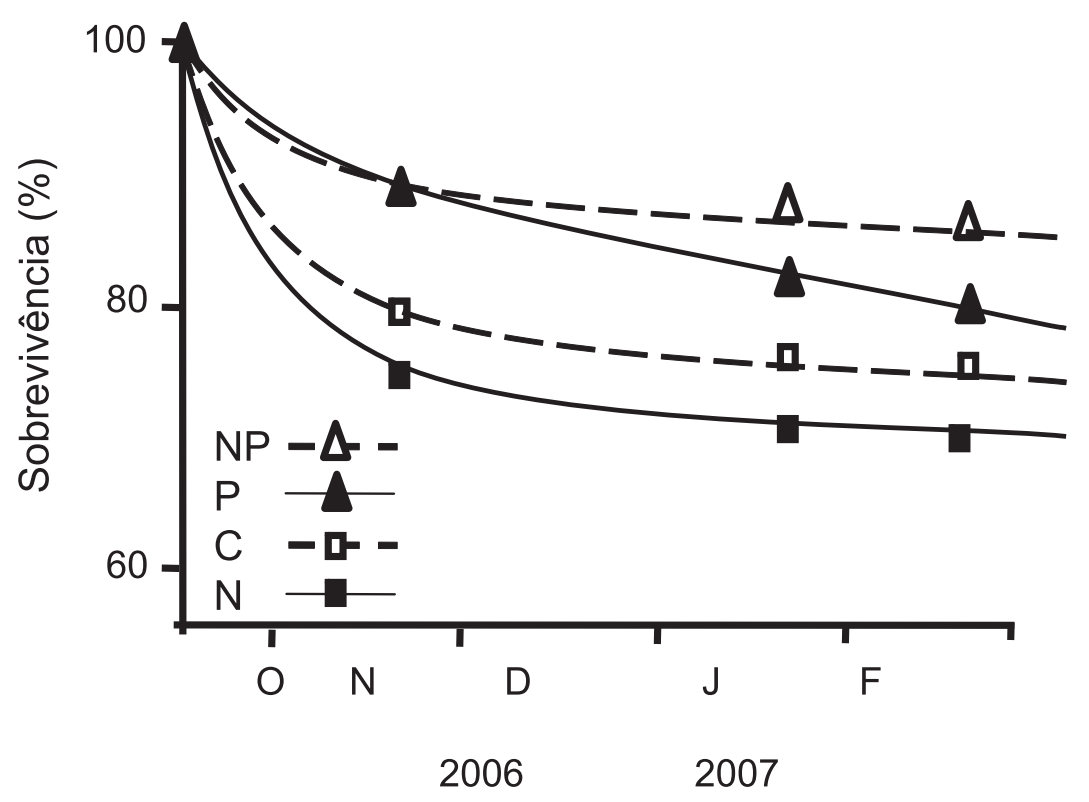

Período de estudo

Figura 5. Curvas de sobrevivência de mudas de S. alterniflora transplantadas no estuário da lagoa dos Patos (RS) submetidas a diferentes tratamentos de adição de nutrientes. Abreviações: $C$, controle sem adição; $N$, nitrogênio; $P$, fósforo; NP, nitrogênio e fósforo.

Figure 5. Survival curves of $S$. alterniflora tillers transplanted in the Patos Lagoon estuary (RS) under different treatments of nutrient addition. Abbreviations: $\mathrm{C}$, control without addition; N, nitrogen; P, phosphorous; NP, nitrogen and phosphorous.

enseada do estuário (irregularmente alagado) da lagoa dos Patos apresentaram alta sobrevivência e um melhor desenvolvimento quando fertilizadas em conjunto com nitrogênio e fósforo (NP). Mudas fertilizadas produziram um maior número de novas hastes, alcançaram maiores alturas e expandiram mais longamente os rizomas sobre o plano entremarés do que as mudas não fertilizadas.

Neste trabalho, foi observada, no primeiro mês, uma taxa de sobrevivência global de $80 \%$. Os primeiros trinta dias de um transplante são críticos para o estabelecimento e, nesse período, são observadas as maiores perdas de mudas. Costa e Marangoni (1997) observaram uma taxa de sobrevivência inicial (primeiro mês) de $56,7 \%$, em plantio realizado no estuário da lagoa dos Patos, mas em localidade onde ocorria intensa pastagem por caranguejos da zona entremarés. Altas mortalidades de transplantes de Spartina também têm sido relacionadas com intensa hidrodinâmica e abrasão por areias grossas (Eleuterius in Kruczynski, 1982; Coultas et al., 1978). Em transplantes de $S$. alterniflora e $S$. patens realizados em estuários macromareais, na Flórida, e micromareais (irregularmente alagados), no Alabama e Mississipi, as sobrevivências variaram de $0 \%$ a $65 \%$ para S. alterniflora e $44 \%$ a $100 \%$ para $S$. patens, de acordo com o espaçamento utilizado no plantio (melhor espaçamento: de 0,3-0,9 m), com o tipo de unidade de propagação (melhor tipo: torrões com solo e várias hastes individuais) e com a época de plantio (melhor época: primavera ou outono) (Eleuterius in Kruczynski, 1982; Coultas et al., 1978; Kruczynski, 1982).

Não foi observada diferença significativa na sobrevivência de plantas de $S$. alterniflora entre os níveis do terreno no estuário da lagoa dos $\mathrm{Pa}$ - tos. Esse resultado contrasta com a maior sobrevivência de mudas de $S$. alterniflora, transplantadas em níveis mais elevados de terrenos entremarés, com menor alagamento, em áreas macromareais (Bertness, 1991; Webb e Dodd, 1989). Bertness (1991) encontrou maiores taxas de sobrevivência em transplantes de $S$. alterniflora e $S$. patens $(85 \%$ e $67 \%$ ) em planos elevados, sujeitos a menor tempo e à freqüência de alagamento, do que em planos mais baixos. Já $S$. densiflora e $S$. maritima, também típicas de marismas, demonstraram sobrevivências de $80-100 \%$ em planos alagados menos de $71 \%$ do tempo $(17 \mathrm{~h}$ por dia), mas não sobreviveram em zona mais baixa do entremarés, alagada $87,5 \%$ do tempo $(21 \mathrm{~h}$ por dia, conforme Castillo et al., 2000). De forma semelhante aos nossos resultados, estudos com $S$. pectinata, que habita banhados de água doce, mostram taxas de sobrevivência variando de 84 a $90 \%$ entre planos altos e baixos (Fraser e Kindscher, 2001; Montemayor et al., 2007). A ausência de influência do nível do terreno na sobrevivência do transplante realizado no estuário da lagoa dos Patos pode estar relacionada à pequena amplitude topográfica entre o nível inferior e superior do plantio, de apenas $7 \mathrm{~cm}$, que gerou uma diferença média na freqüência de alagamento das mudas, nesses extremos, de apenas $19 \%$.

Não foi observada diferença significativa na sobrevivência de plantas de $S$. alterniflora entre os tratamentos de adição de nutrientes no estuário da lagoa dos Patos. Esse resultado contrasta com os dados de alguns estudos que mostram aumento na taxa de sobrevivência de $S$. foliosa, quando as plantas foram tratadas com composto de feófitas gigantes (kelps) (O’Brien e Zedler, 2006). Esses autores caracterizaram tal composto como rico em nitrogênio orgânico e inorgânico, mas não quantificaram a quantidade de fósforo. Resultados semelhantes aos encontrados neste estudo não mostram diferenças significativas na sobrevivência de mu- 


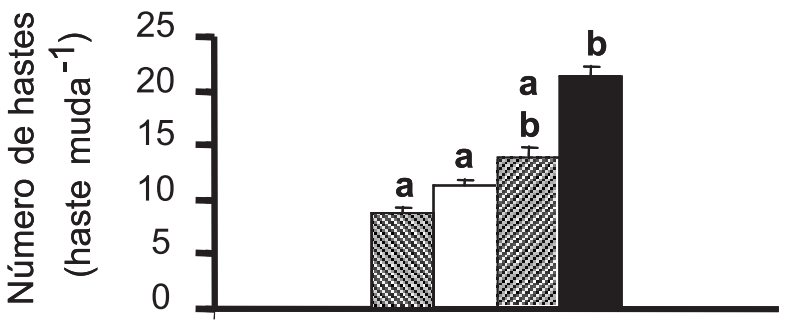

A

B

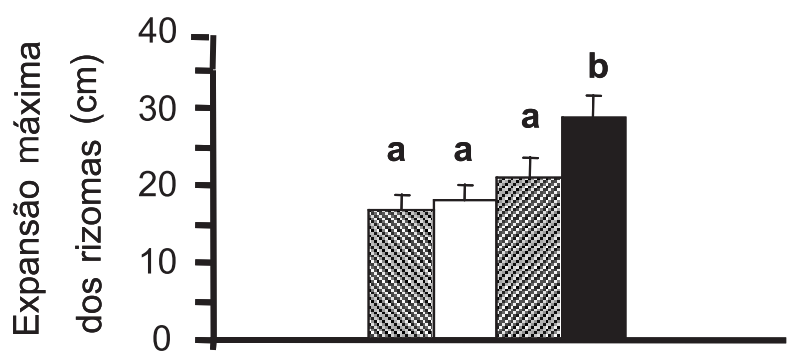

C
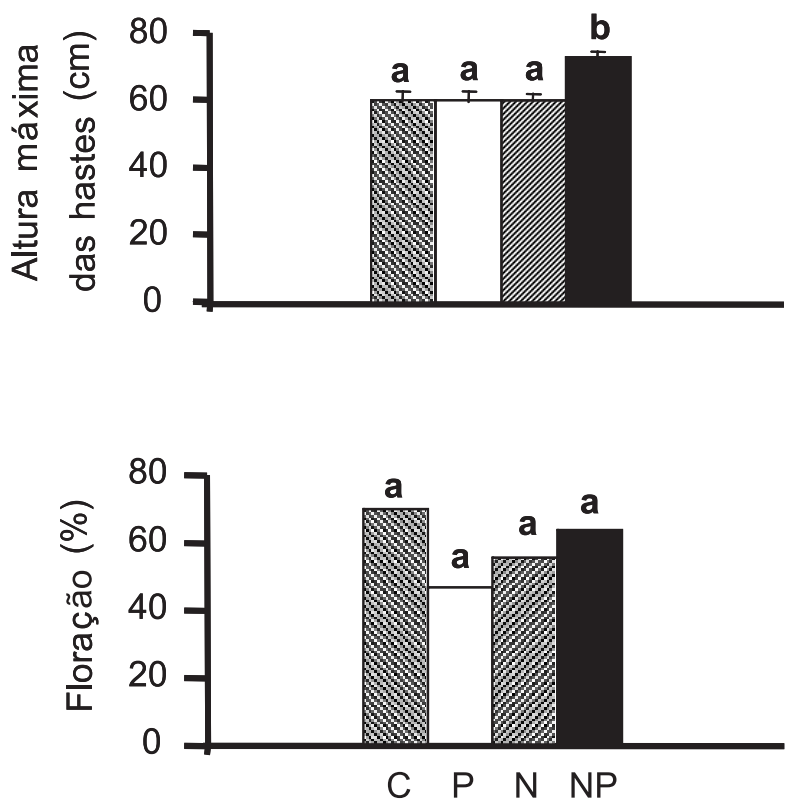

Figura 6. Médias globais ( \pm erro-padrão) do número de hastes produzidas (A), expansão de rizomas (B), altura máxima (C) e porcentagem de hastes que floresceram (D) de $S$. alterniflora após quatro meses de plantio e adição de nutrientes no estuário da lagoa dos Patos (RS). Diferentes letras minúsculas indicam médias significativamente diferentes $(p<0,05)$, conforme os testes de Tukey $(\mathbf{A}-\mathbf{C})$ e Qui-quadrado (D). Abreviações: C, controle sem adição; $N$, nitrogênio; $P$, fósforo; NP, nitrogênio e fósforo.

Figure 6. Global average ( \pm standard-error) of the number of produced tillers (A), maximum horizontal expansion of rhizomes $(\mathbf{B})$, maximum tiller height $(\mathbf{C})$ and percentage of flowering tillers (D) of $S$. alterniflora, after 4 months of planting and nutrient additions in the Patos Lagoon estuary (RS). Different lower case letters indicate significant differences $(p<0.05)$ between treatment levels according Tukey test $(\mathbf{A}-\mathbf{C})$ and Chi-square test (D). Abbreviations: C, control without addition; N, nitrogen; P, phosphorous; NP, nitrogen and phosphorous. das de Spartina, quando tratadas com nutrientes (Webb e Dodd, 1989).

Após três meses de adição conjunta de $\mathrm{N}$ e $\mathrm{P}$, ocorreram aumentos médios de $143 \%$ no número de hastes por propágulo, 21\% na altura das hastes e $75 \%$ na expansão horizontal dos rizomas das mudas de S. alterniflo$r a$, em relação às mudas do controle sem adição de nutrientes. Broome et al. $(1975,1983)$ encontraram também um melhor desenvolvimento de $S$. alterniflora quando as plantas foram fertilizadas com nitrogênio e fósforo juntos. As mudas de S. alterniflora tratadas no presente estudo apenas com nitrogênio apresentaram um ganho de $54 \%$ no número de hastes e de $22 \%$ na expansão horizontal. Estudos anteriores mostraram um melhor crescimento de plantas de marismas, principalmente da biomassa aérea, pela presença natural de maior quantidade de nitrogênio no sedimento (Smart e Barko, 1980) ou como resposta à adição de diferentes formas de nitrogênio (Buresh et al., 1980; Long e Manson, 1983; Mendelssohn, 1979; Smart, 1982; Valiela e Teal, 1974). Valiela e Teal (1974) encontraram aumentos significantes no crescimento de $S$. alterniflora, S. patens e Distichlis spicata, quando fertilizadas com nitrogênio na forma de uréia. O'Brien e Zedler (2006) encontraram aumentos de $47 \%$ para número de hastes de $S$. foliosa com adição de composto orgânico rico em nitrogênio. Marangoni (2001) observou em plantas de $S$. densiflora tratadas apenas com cloreto de amônio aumentos de $203 \%$ no número de hastes, $40 \%$ na altura da haste e $30 \%$ da extensão horizontal, em relação aos tratados com água.

A marcada resposta de crescimento observada nas mudas de S. alterniflo$\mathrm{ra}$, quando fertilizadas com NP, ocorreu em um local considerado eutrofizado (Saco da Mangueira), devido a um grande aporte de efluentes domésticos (ricos em matéria orgânica) e industriais de fábricas de fertilizantes. Concentrações médias de nitrogênio na forma de amônio, variando de 127 

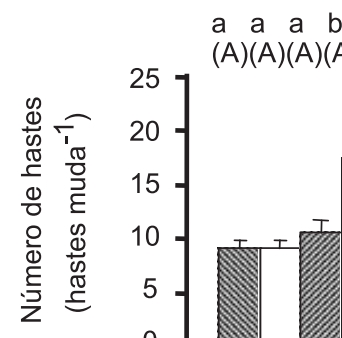

$(A)(A)(A)(A)$

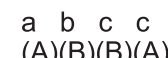

T-

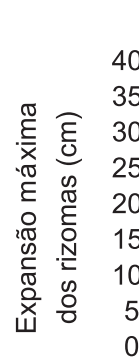
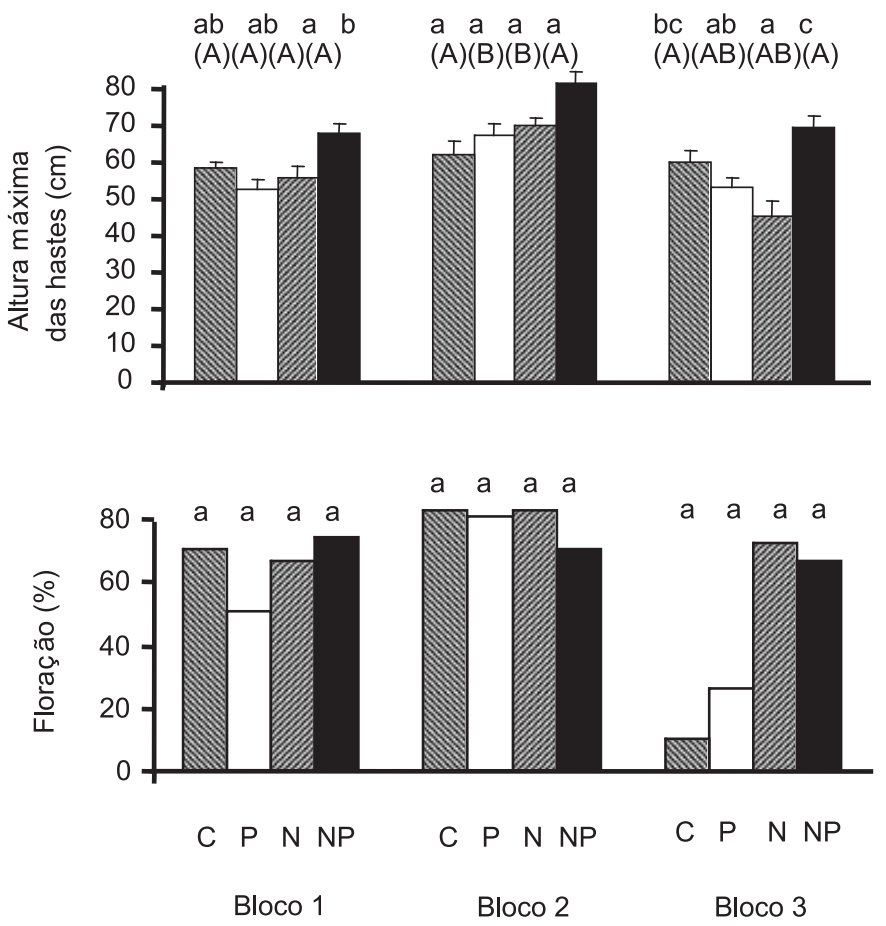

Figura 7. Médias por blocos de plantio ( \pm erro-padrão) do número de hastes produzidas (A), expansão de rizomas (B), altura máxima (C) e porcentagem de hastes que floresceram (D) de S. alterniflora após quatro meses de plantio e adição de nutrientes. Diferentes letras minúsculas indicam médias significativamente $(p<0,05)$ diferentes entre tratamentos de um mesmo bloco. Diferentes letras maiúsculas indicam médias significativamente $(p<0,05)$ diferentes entre blocos para adição dos mesmos nutrientes. Significâncias estimadas pelos testes de Tukey (A-C) e Chi-quadrado (D). Abreviações: C, controle sem adição; N, nitrogênio; $P$, fósforo; NP, nitrogênio e fósforo.

Figure 7. Average by planting blocks ( \pm standard-error) of the number of produced tillers $(\mathbf{A})$, maximum horizontal expansion of rhizomes (B), maximum tiller height (C) and percentage of flowering tillers (D) of $S$. alterniflora, after 4 months of growth and nutrient additions in the Patos Lagoon estuary (RS). Different lower case letters indicate significant differences $(p<0.05)$ between treatment levels of the same block. Different capital letters indicate significant differences $(p<0.05)$ between blocks for the same nutrients. Significances tested by Tukey test (A-C) and Chi-square test (D). Abbreviations: C, control without addition; N, nitrogen; P, phosphorous; NP, nitrogen and phosphorous. a $393 \mu \mathrm{M}$, e de fósforo na forma de fosfato, entre 4,4 e 59,3 $\mu \mathrm{M}$, foram registradas na água intersticial do sedimento superficial $(0-13 \mathrm{~cm})$ do Saco da Mangueira (Baumgarten et al., 2001, 2005). Plantas de S. alterniflora e outras espécies dominantes de marismas do Saco da Mangueira apresentam maiores concentrações intracelulares de $\mathrm{N}$ do que outras plantas de enseadas não-eutrofizadas (Abreu et al., 2006). Esse resultado demonstra que, mesmo em ambientes de alta produtividade, com disponibilidade de macronutrientes, $S$. alterniflora apresenta uma alta demanda de nitrogênio e fósforo para atingir máximas taxas de crescimento e produtividade. Resultados semelhantes de alta demanda de nutrientes de $S$. alterniflora foram também encontrados por outros autores (Adam, 1990; Broome et al., 1975; Levine et al., 1998; Smart e Barko, 1980; Valiela et al., 1974).

A resposta de crescimento de $S$. alterniflora frente à adição exclusiva de $\mathrm{N}$ e de $\mathrm{P}$ variou espacialmente entre níveis e blocos de plantio. Apesar da ausência de diferenças na granulometria e no teor de matéria orgânica no sedimento entre blocos, que poderiam indicar uma variação espacial no status nutricional, variações significativas na concentração de fosfato foram observadas entre níveis do plantio. Plantas no nível médio de plantio apresentaram maiores desempenhos em todos os parâmetros de crescimento. Plantas do bloco central, quando tratadas com $\mathrm{N}$, apresentaram maior número de hastes e expansão dos rizomas e, quando tratadas com $\mathrm{P}$, produziram hastes mais altas do que as plantas dos demais blocos tratadas com os mesmos nutrientes. Adicionalmente, apenas no bloco central parece ter ocorrido limitação do crescimento por $\mathrm{P}$, visto que a adição deste elemento provocou aumento significativo de um parâmetro de crescimento (número de hastes) em relação às plantas do controle sem adição de nutrientes. $\mathrm{O}$ melhor desempenho das plantas no nível médio, particularmente no bloco 
A central, e as diferentes respostas, entre blocos, à adição de nutrientes parecem estar relacionados à ação biogênica de S. alterniflora, modificadora das condições físico-químicas do sedimento. Os melhores crescimentos das plantas no nível médio e no bloco central de plantio estão relacionados às interações e feedbacks entre o tamanho das plantas, a concentração de oxigênio, B a disponibilidade de nutrientes e a toxicidade de elementos abundantes nas marismas, como sulfetos, que afetam a assimilação de nutrientes por S. alterniflora (Smart e Barko, 1980; Morris, 1984; Adam, 1990). Tanto $S$. alterniflora e $S$. patens (Bertness, 1991), como $S$. maritima e $S$. densiflora (Castillo et al., 2000) demonstraram melhores taxas de crescimento com o aumento do nível do terreno, onde os estresses devido ao alagamento são reduzidos. Sob condições anaeróbicas, por exemplo, S. alterniflora e $S$. patens diminuem a absorção de amônio (Morris, 1984). Quanto mais crescem e ocupam o sedimento, espécies de Spartina aumentam o transporte de ar rico em oxigênio para sua rizosfera, por meio de um sistema aerenquimatoso bem desenvolvido em suas hastes, rizomas e raízes, bem como aceleram

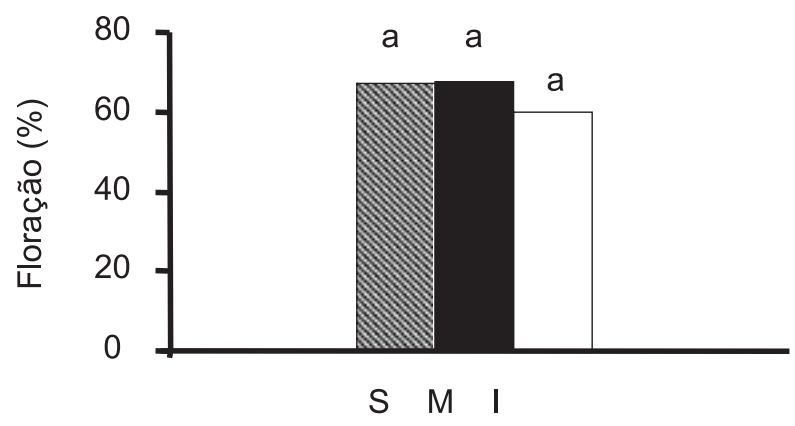

Níveis do terreno

Figura 8. Médias ( \pm erro-padrão) de todos tratamentos de adição de nutrientes, agregadas por níveis de plantio, do número de haste produzidas $(\mathbf{A})$, expansão de rizomas $(\mathbf{B})$, altura máxima (C) e porcentagem de hastes que floresceram (D) de S. alterniflora após quatro meses de crescimento no estuário da lagoa dos Patos (RS). Diferentes letras minúsculas indicam médias significativamente $(p<0,05)$ diferentes conforme os testes de Tukey $(\mathbf{A}-\mathbf{C})$ e Qui-quadrado (D). Abreviações: S, nível superior; M, nível médio; I, nível inferior.

Figure 8. Average values ( \pm standard-error) of all nutrient treatments aggregated by intertidal levels of planting of the number of produced tillers (A), maximum horizontal expansion of rhizomes (B), maximum tiller height (C) and percentage of flowering tillers (D) of S. alterniflora, after 4 months of growth in the Patos Lagoon estuary (RS). Different lower c ase letters indicate significant differences $(p<0.05)$ between treatment levels according Tukey test $(\mathbf{A}-\mathbf{C})$ and Chi-square test (D). Abbreviations: S, upper level; M, medium level; I, lower level. a deposição e fixação de sedimento, alterando as condições ambientais a seu favor (Jones et al., 1994; Pennings e Bertness, 2001). Essa capacidade de S. alterniflora oxigenar o sedimento e aumentar o potencial redox está diretamente relacionada ao tamanho da área vegetada (Bertness, 1991), ao tamanho das plantas e à densidade de hastes (Proffit et al., 2003). Assim, as plantas no nível médio e, particularmente, no bloco central do plantio, estão sujeitas a um ambiente físico-químico mais favorável ao seu desenvolvimento, fortemente influenciado por seus vizinhos, que amenizam a toxidez, inibindo a formação de sulfetos e metano, mas também afetam a disponibilidade de nutrientes como o fosfato à medida que a marisma é colonizada (Metclafe et al., 1986; Pennings e Bertness, 2001). Além de uma maior demanda de nu- 


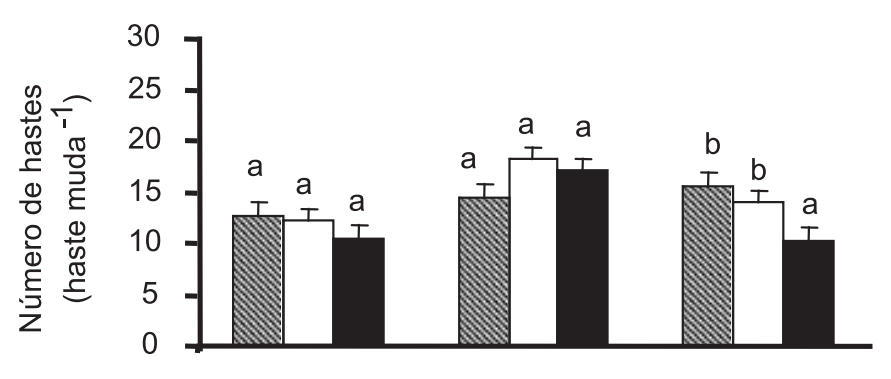

B

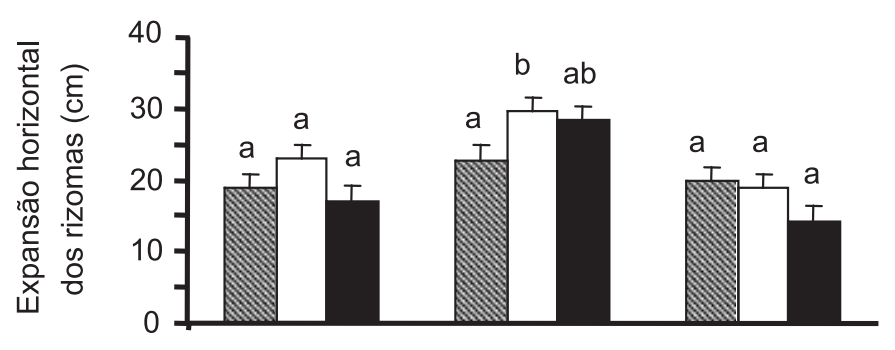

C

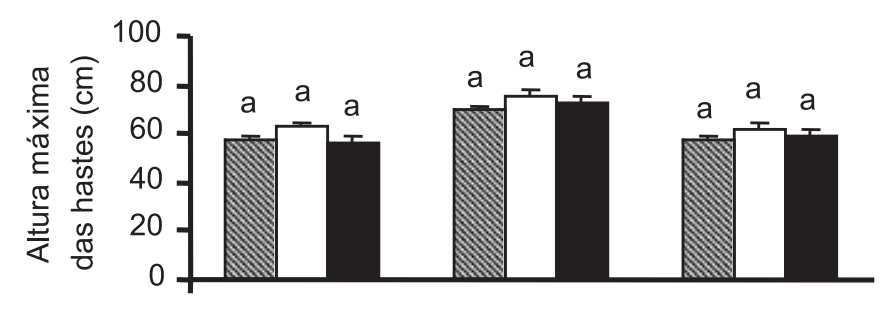

D

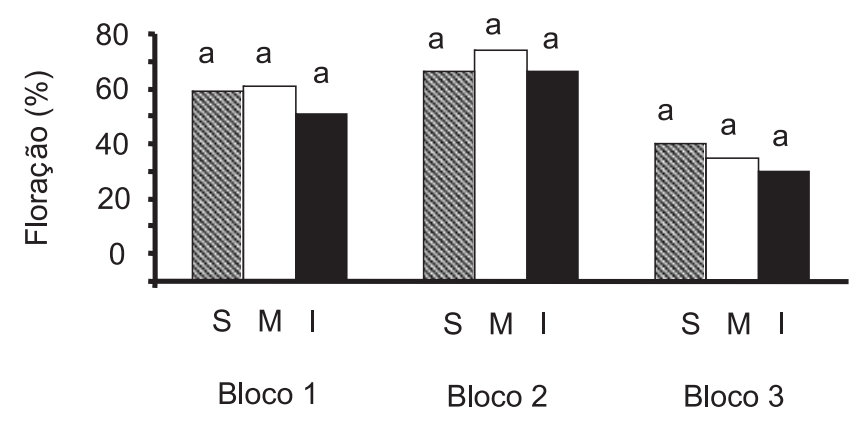

Figura 9. Médias ( \pm erro-padrão) de todos tratamentos de adição de nutrientes, agregadas por níveis e blocos de plantio, do número de hastes produzidas (A), expansão de rizomas (B), altura máxima (C) e porcentagem de hastes que floresceram (D) de S. alterniflora após quatro meses de crescimento no estuário da lagoa dos Patos (RS). Diferentes letras minúsculas indicam médias significativamente $(p<0,05)$ diferentes entre os níveis no mesmo bloco conforme os testes de Tukey (A-C) e Qui-quadrado (D). Abreviações: S, nível superior; $M$, nível médio; I, nível inferior.

Figure 9. Average values ( \pm standard-error) of all nutrient treatments aggregated by intertidal levels and blocks of planting of the number of produced tillers $(\mathbf{A})$, maximum horizontal expansion of rhizomes (B), maximum tiller height (C) and percentage of flowering tillers (D) of S. alterniflora after 4 months of growth in the Patos Lagoon estuary (RS). Different lower case letters indicate significant differences $(p<0.05)$ between treatment levels according Tukey test $(\mathbf{A}-\mathbf{C})$ and Chi-square test (D). Abbreviations: S, upper level; M, medium level; I, lower level. trientes, outra conseqüência do melhor crescimento das plantas de $S$. alterniflora no bloco central é uma forte alteração do status local de nutrientes, que pode limitar o crescimento por fósforo. A adição de apenas nitrogênio resulta em maior crescimento de S. alterniflora, mas maiores densidades de hastes de plantas mais altas geram a necessidade de mais nutrientes. Broome et al. (1975) demonstraram que a disponibilidade de fósforo rapidamente se torna limitante, quando a quantidade de nitrogênio adicionada a plantios aumenta. Já a Spartina requer muito nitrogênio em relação ao fósforo, e sua relação N/P, sob condições de mínima necessidade para o crescimento, é de 16,1:1 (Smart e Barko, 1980). Conforme esses mesmos pesquisadores, essa relação para plantas emergentes de água doce é de 7,5:1. Planos de lama entremarés não vegetados do estuário da lagoa dos Patos possuem baixo teor de nitrogênio disponível para as plantas e um alto teor de fosfato, sendo observadas relações N/P tão baixas quanto 2,5:1 (Marangoni, 2001). A relação N/P, contudo, vai aumentando à medida que $S$. alterniflora coloniza o plano de lama, incorpora matéria orgânica no sedimento e soergue o terreno. $\mathrm{O}$ aumento do potencial redox, devido à colonização por $S$. alterniflora e à ação do seu aerênquima, permite a presença de ferro oxidado e óxido de alumínio, que fixam o fosfato, tornando esse nutriente menos disponível no sedimento (Baumgarten et al., 2001; Broome et al., 1983; Esteves, 1988; Marangoni, 2001). Assim, áreas mais densamente vegetadas no nível médio do bloco central respondem marcadamente à adição de $\mathrm{N}$ e $\mathrm{P}$, enquanto áreas periféricas do plantio não possuem as mesmas condições ambientais, por não terem sido ainda alteradas por essa espécie bioengenheira.

Os resultados do estudo de adição de nutrientes demonstram uma contribuição significativa da disponibilidade de fósforo para a sobrevivência de mudas de $S$. alterniflora na fase inicial de co- 
lonização dos planos de lama do estuário da lagoa dos Patos. As plantas dessa espécie mostram rápida formação de novas hastes e alongamento de seus rizomas sob planos de lama com a adição de nitrogênio. Entretanto, o status destes nutrientes e as interações das respostas de $S$. alterniflora a eles são dependentes das alterações físicoquímicas realizadas pelas próprias plantas, à medida que formam novas áreas de marismas.

\section{Agradecimentos}

Autores agradecem ao Programa Costa Sul/Banco Interamericano de Desenvolvimento pelo financiamento do projeto. A bolsa de mestrado de P.T.M. foi concedida pela CAPES. O $\mathrm{CNPq}$ apoiou este projeto através da bolsa de pesquisa de C.S.B.C.

\section{Referências}

ABREU, P.C.; COSTA, C.S.B.; BEMVENUTI, C.E.; ODEBRECHT, C.; GRANÉLI, W.; ANESIO, A.M. 2006. Eutrophication processes and trophic interactions in a shallow estuary: Preliminary results based on stable isotope analysis (d13C and $\mathrm{d} 14 \mathrm{~N})$. Estuaries and Coasts, 29:279-287.

ADAM, P. 1990. Saltmarsh Ecology. New York, Cambridge University Press, $461 \mathrm{p}$.

ADAM, P. 2002. Saltmarshes in a time of change. Environmental Conservation, 29(1):3961 .

BAUMGARTEN, M.G.Z.; ROCHA, J.M.B; NIENCHESKI, L.F.H. 1996. Manual de análises em Oceanografia Química. Rio Grande, Editora Furg, 143 p.

BAUMGARTEN, M.G.Z:; NIENCHESKI, L.F.H.; VEECK, L. 2001. Nutrientes na coluna da água e na água intersticial de sedimentos de uma enseada rasa estuarina com aportes de origem antrópica (RS- Brasil). Atlântica, 23:101-116.

BAUMGARTEN, M.G.Z.; NIENCHESKI, L.F.H.; MARTINS, B.A.D. 2005. Saco do Justino (RS-Brasil): amônio e fosfato na coluna d' água e na água intersticial de uma enseada não contaminada. Atlântica, 27(2):113-129.

BEEFTINK, W.G. 1977. Salt-marshes. In: R.S.K. BARNES (ed.), The coastline. New York, Wiley, p. 93-121.

BERTNESS, M.D. 1991. Zonation of Spartina patens and Spartina alterniflora in New England salt marsh. Ecology, 72:138-148.

BROOME, S.W.; SENECA, E.D.; WOODHOUSE, W.W. 1981. Planting marsh grasses for erosion control. Raleigh, UNC Sea Grant College Publication UNC-SG- 81-09, 11 p. BROOME, S.W.; SENECA, E.D.; WOODHOUSE, W.W. 1983. The effects of source, rate and placement of nitrogen and phosphorus fertilizers on growth of Spartina alterniflora transplants in North Carolina. Estuaries, 6(3):212-226.

BROOME, S.W.; WOODHOUSE, W.W.; SENECA, E.D. 1975. The relationship of mineral nutrients to growth of Spartina alterniflora in North Carolina: II. The effects of N, P, and Fe fertilizers. Soil Science Society of America Proceedings, 39(2):201-207.

BURESH, R.J.; DeLAUNE, R.D.; PATRICK, W.H.Jr., 1980. Nitrogen and phosphorus distribution and utilization by Spartina alterniflora in a Louisiana Gulf Coast marsh. Estuaries, 3(2): $111-121$

CASTILLO, J.M; FERNÁNDEZ-BACO, L.; CASTELLANOS, E.M.; LUQUE, C.J.; FIGUEROA, M.E.; DAVY, A.J. 2000. Lower limits of Spartina densiflora and S. maritima in a Mediterranean salt marsh determined by diferent ecophysiological tolerances. Journal of Ecology, 88:801-812.

CHALMES, A.G. 1979. The effects of fertilization on nitrogen distribution in a Spartina alterniflora salt marsh. Estuarine and Coastal Marine Science, 8:327-337.

CHUNG, C. 1982. Low marshes, China. In: R.R. LEWIS (ed.), Creation and restoration of coastal plant communities. Boca Raton, CRC Press, p. 131-146.

CHUNG, C. 1993. Thirty years of ecologica engineering with Spartina plantations in China. Ecological Engineering, 2:261-289.

COIMBRA, F.L.; COSTA, C.S.B. 2006. Mapeamento digital dos macrohabitats de dunas e marismas da costa do Rio Grande do Sul através de imagens de satélite e fotografias aéreas. In ENCONTRO NACIONAL DE GERENCIAMENTO COSTEIRO - ENCOGERCO 2006, Florianópolis, Agência Brasileira de Gerenciamento Costeiro, Anais, Trabalho 9, p. 1-4.

CORPERTINO, M.; COSTA, C.S.B.; SEELIGER, U. 1997. Dinâmica populacional de Spartina alterniflora em pântanos salgados do estuário da Lagoa dos Patos, Rio Grande, RS In: J.E. SANTOS (ed.), Anais do VIII Seminário Regional de Ecologia. São Carlos, Programa de Pós-Graduação em Ecologia e Recursos Renováveis - UFSCar, p. 295-312.

COSTA, C.S.B. 1998. Marismas irregularmente alagadas. In: U. SEELIGER; C. ODEBRECHT; J.P. CASTELLO (eds.), Os ecossistemas costeiro e marinho do extremo Sul do Brasil. Rio Grande, Editora Ecoscientia, p. 82-87.

COSTA, C.S.B. 2009. Restoration of coasta habitats in Brazil using native salt marsh plants. In: S. GREIPSSON (ed.), Restoration Ecology: $A$ notebook. Sudbury, Jones and Bartlett Publishers (no prelo).

COSTA, C.S.B.; DAVY, A.J. 1992. Coasta saltmarsh communities of Latin America. In: $\mathrm{U}$.
SEELIGER (ed.). Coastal plant communities of Latin America. New York, Academic Press, p. 179-198.

COSTA, C.S.B.; MARANGONI. J.C. 1997. Criação de marismas com espécies nativas: experimento piloto no sul do Brasil. In: CONGRESSO LATINO AMERICANO DE CIÊNCIAS DO MAR, 7, Santos, Anais, 1:200-201.

COSTA, C.S.B.; MARANGONI, J.C.; AZEVEDO, A.M.G. 2003. Plant zonation in irregularly flooded salt marshes: relative importance of stress tolerance and biological interactions. Journal of Ecology, 91(6):951-965.

COSTA, C.S.B.; SEELIGER, U.; KINAS, P.G. 1988. The effect of wind velocity and direction on the salinity regime in the lower Patos Lagoon estuary. Ciência e Cultura, 40(9):909-912

COSTA, C.S.B.; SEELIGER, U.; OLIVEIRA, C.P.L.; MAZO, A.M.M. 1997. Distribuição, funções e valores das marismas e pradarias submersas no estuário da Lagoa dos Patos (RS, Brasil). Atlântica, 19:65-83.

COULTAS, C.L.; BREITENBECK, G.A.; KRUCZYNSKI, W.L.; SUBRAHMANYAM, C.B. 1978. Vegetative stabilization of dredge spoil in North Florida. Journal of Soil and Water Conservation, 33(4):183-185.

CUNHA, S.R.; ASMUS, M.; COSTA, C.S.B. 2005. Production dynamics of Spartina alterniflora salt marshes in the estuary of the Patos Lagoon (RS, Brazil): A simulation model approach. Brazilian Journal of Aquatic Science and. Technology, 9(2):75-85.

ESTEVES, F.A. 1988. Fundamentos de Limnologia. Rio de Janeiro, Editora Interciência FINEP, $575 \mathrm{p}$.

FRASER, A.; KINDSCHER, K. 2001. Tree spade transplanting of Spartina pectinata (Link) and Eleocharis macrostachya (Britt.) in a prairie wetland restoration site. Aquatic Botany, 71:297-304.

ISACCH, J.P.; COSTA, C.S.B.; RODRÍGUEZ-GALLEGO, L.; CONDE, D.; ESCAPA, M.; GAGLIARDINI, D.A.; IRIBARNE, O.O. 2006. Distribution of salt marsh plant communities associated with environmental factors along a latitudinal gradient on the southwest Atlantic coast. Journal of Biogeography, 33(5):888-900.

JONES, C.G.; LAWTON, J.H.; SHACHAK, M. 1994. Organisms as ecosystem engineers. Oikos, 69:373-386.

KNUTSON, P.L.; WOODHOUSE, W.W. 1982. Pacific coastal marshes. In: R.R. LEWIS (ed.), Creation and restoration of coastal plant communities. Boca Raton, CRC Press, p. 111-130.

KRUCZYNSKI, W.L. 1982. Salt marshes of the northeastern Gulf of Mexico. In: R.R. LEWIS (ed.), Creation and restoration of coastal plant communities. Boca Raton, CRC Press, p. 71-88.

LEVINE, J.M.; BREWER, J.S.; BERTNESS, M.D. 1998. Nutrients, competition and plant 
zonation in a New England salt marsh. Journal of Ecology, 86:285-292.

LEWIS, R.R. 1982. Low marshes, peninsular Florida. In: R.R. LEWIS (ed.), Creation and restoration of coastal plant communities. Boca Raton, CRC Press, p. 147-154.

LONG, S.P.; MANSON, C.F. 1983. Saltmarsh ecology. Tertiary Level Biology Series. Glasgow, Blackie, p. 180.

MARANGONI, J.C. 2001. Interação competitiva e habilidade colonizadora das plantas dominantes de uma marisma irregularmente inundada no estuário da Lagoa dos Patos (RS, Brasil). Dissertação de Mestrado, Universidade Federal do Rio Grande - FURG, Rio Grande, 169 p.

MARANGONI, J.C. 2008. Subsídios para o gerenciamento das marismas no estuário da Lagoa dos Patos (RS). Tese de Doutorado, Universidade Federal do Rio Grande - FURG, Rio Grande, $141 \mathrm{p}$.

MATTHEWS, G.A.; MINELLO, T.J. 1994.

Technology and success in restoration, creation, and enhancement of Spartina alterniflora marshes in the United States. NOAA Coastal Ocean Program Decision Analysis Series, No. 2. Silver Spring, NOAA Coastal Ocean Office, $17 \mathrm{p}$.

MENDELSSOHN, I.A. 1979. The influence of nitrogen level, form and application method on the growth response of Spartina alterniflora in North Carolina. Estuaries, 2:106-112.

METCALFE, W.S.; ELLISON, A.M.; BERTNESS, M.C. 1986. Survivorship and spatial development of Spartina alterniflora Loisel. (Gramineae) seedings in a New England salt marsh. Annals of Botany, 58:249-258.

MONTEMAYOR, M.B.; PRICE, J.S.; ROCHEFORT, L.; BOUDREAU, S. 2007. Tempo- ral variations and spatial patterns in saline and waterlogged peat fields: 1 - Survival and growth of salt marsh graminoids. Environmental and Experimental Botany, 62:333-342.

MORRIS, J.T. 1984. Effects of oxygen and salinity on ammonium uptake by Spartina alterniflora Loisel. and Spartina patens (Aiton) Muhl.. Journal of Experimental Marine Biology and Ecology, 78:87-98.

O'BRIEN. E.; ZEDLER, J.B. 2006. Accelerating the restoration of vegetation in a southern California salt marsh. Wetlands Ecology and Management, 14:269-286.

ODUM, W.E. 1988. Comparative ecology of tidal freshwater and salt marshes. Annual Review of Ecology and Systematics, 19:146-176.

PENNINGS, S.C.; BERTNESS, M.D. 2001. In: M.D. BERTNESS; S.D. GAINES; M.E. HAY (eds.), Salt marsh communities. Sunderland, Sinauer Associates, p. 289-316.

PROFFIT, C.E.; TRAVIS, S.E.; EDWARDS, K.R. 2003. Genotype and elevation influence Spartina alterniflora colonization and growth in a created salt marsh. Ecological Applications, 13(1): 180-192.

SEELIGER, U.; COSTA, C.S.B. 1998. Impactos naturais e humanos. In: U. SEELIGER; C. ODEBRECHT; J.P. CASTELLO (eds.), Os ecossistemas costeiro e marinho do extremo Sul do Brasil. Rio Grande, Editora Ecoscientia, p. 217-226.

SIEGEL, S. 1975. Estatística não-paramétrica para ciências do comportamento. São Paulo, McGraw-Hill do Brasil Ltda, p. 350.

SMART, R.M. 1982. Distribution and environmental control of productivity and growth form of Spartina alterniflora (Loisel.). In: D.N. SEN; K.S. RAJPUROHIT (eds.), Contributions to the ecology of halophytes. Boston, Dr. W. Junk Publishers, p. 127-142.

SMART, R.M.; BARKO, J.W., 1980. Nitrogen nutrition and salinity tolerance of Distichlis spicata and Spartina alterniflora. Ecology, 61(3):630-638.

VALIELA, I.; TEAL, J.M., 1974. Nutrient limitation in salt marsh vegetation. In: R.J. REINOLD; N.H. QUEEN (eds.), Ecology of halophytes. Londres, Academic Press, Inc., p. 547-563.

VALIELA, I.; TEAL, J.M., 1979. Inputs, outputs and interconversions of nitrogen in a salt marsh ecosystem. In: R.L. JEFFERIES; A.J. DAVY (eds.), Ecological processes in coastal environments. Londres, Blackwell Scientific Publications, p. 399-414.

WEEB, J.W.Jr. 1982. Salt marshes of the western Gulf of Mexico. In: R.R. LEWIS (ed.), Creation and restoration of coastal plant communities. Boca Raton, CRC Press, p. 89-110.

WEBB, J.W.Jr.; DODD, J.D. 1989. Spartina alterniflora response to fertilizer, planting dates, and elevation in Galveston bay, Texas. Wetlands, 9:61-72.

WOODHOUSE, W.W.; KNUTSON, P.L. 1982. Atlantic Coastal marshes In: R.R. LEWIS (ed.), Creation and restoration of coastal plant communities. Boca Raton, CRC Press, p. 45-70.

ZAR, J.H. 1984. Biostatistical analysis. Nova Jersey, Prentice-Hall Inc., 718 p.

Submitted on March 28, 2008 Accepted on August 18, 2008.

148 | Volume 3 number 3 september - december 2008 